\title{
Thermal Conductivity of Square-Well Fluids
}

\author{
R. SRIVASTAVA And K.N. Khanna* \\ Department of Physics, V.S.S.D. College, Kanpur, India \\ (Received June 29, 2009; in final form December 1, 2009)
}

\begin{abstract}
The thermal conductivity of a system consisting of square-well particles has been determined by the extension of the Enskog formula of the hard-sphere model to square-well fluid. The approach is the same as applied for the diffusion coefficients and shear viscosity of square-well fluid. The addition of an attractive part in the hard-sphere potential such as square-well potential remains insensitive to influence the thermal conductivity. The results obtained are in a good agreement with the molecular dynamics results.
\end{abstract}

PACS numbers: $66.25 .+\mathrm{g}$

\section{Introduction}

The knowledge of the transport coefficients such as shear viscosity, self-diffusion coefficients, and thermal conductivity of liquids is indispensable for the understanding of industrial applications. Their molecular theoretical calculations are the principle aims of kinetic theory and non-equilibrium dynamics. While the transport properties at low densities are well understood through the Chapman-Enskog theory [1] of the hard-sphere (HS) system, the dense hard fluids are far from being understood. Dense hard-spheres possess enough attributes of many-body systems and are understood with kinetic and mode coupling theories [2-4]. There are very few theoretical studies reported in the literature for thermal conductivity of dense fluids. However, molecular dynamic simulation results for simple fluids are available in the literature [5-9] for hard-sphere system and Lennard-Jones fluids. Heyes and Powles [10] have calculated thermal conductivity of steeply repulsive potential (SRP) in which the particles interact with a potential $\phi(r)=\varepsilon(\sigma / r)^{n}$ where $n=\infty$ represents the hard-sphere system. Michels and Trappeniers [11] have calculated the thermal conductivity of the square-well fluids by means of molecular dynamical computer simulations.

In the present work, thermal conductivity of a square-well fluid has been determined by the application of improved Enskog formula [12, 13] of a hard-sphere system. The hard-sphere system has been transformed into a square-well system by changing appropriate pair-correlation function at contact. This method was found successful in the determination of diffusion coefficients and shear viscosity of a square-well fluid, as described in our previous works $[14,15]$.

\section{Theory}

The square-well fluid is an excellent model for a liquid in which the internal degrees of freedom of the individual

\footnotetext{
* corresponding author; e-mail: knkhanna@rediffmail.com
}

atoms are not important. The pair potential for a square-well fluid is defined as:

$$
\phi(r)= \begin{cases}\infty, & r \leq \sigma, \\ -\varepsilon, & \sigma<r<\lambda \sigma, \\ 0, & r \geq \lambda \sigma,\end{cases}
$$

where $r$ is the radial coordinate, $\sigma$ is the diameter of hard core, $\lambda \sigma$ is the diameter of the surrounding well and $\varepsilon$ is the magnitude of attractive part of the potential. The well width is considered as $\lambda=1.5$ throughout this paper.

Consider a collective property transport coefficient $X$ representing either bulk or shear viscosity, or the thermal conductivity. The Green-Kubo formula for this quantity is

$$
X=A \int_{0}^{\infty}\left\langle B\left(s^{\prime}+1\right) B\left(s^{\prime}\right)\right\rangle_{s^{\prime}} \mathrm{d} t
$$

where $\langle\cdots\rangle_{s^{\prime}}$ is a time correlation function averaged over a sampling time $s^{\prime}$ which is also the simulation time in practice. The property $B$ would be the shear stress for the shear viscosity, $P(t)-\langle P\rangle$ for the bulk viscosity $[P(t)$ is the instantaneous pressure and $\langle P\rangle$ is the average pressure], or the heat flux for the thermal conductivity. The constant $A$ is a simple function of numerical prefactors and basic constants such as the volume of the system $(V)$, the temperature $T$, and Boltzmann's constant $k_{\mathrm{B}}$. It is convenient to define a normalized time correlation function

$$
C(t)=\left\langle B\left(s^{\prime}+t\right) B\left(s^{\prime}\right)\right\rangle_{s^{\prime}} /\left\langle B^{2}\left(s^{\prime}\right)\right\rangle_{s^{\prime}} .
$$

Substitution of Eq. (3a) in Eq. (2) gives

$$
X=C_{\infty} \int_{0}^{\infty} C(t) \mathrm{d} t
$$

where

$$
C_{\infty}=A\left\langle B^{2}\right\rangle .
$$

For the heat flux correlation function, $C_{\infty}=M_{\infty}$, the so-called "thermal modulus" which was derived in Ref. [10]. The time correlation function $C(t)$ can be decomposed into three separate functions, one derived entirely from the interaction potential, second which is purely kinetic, and another that is a mix of kinetic and interaction parts. For thermal conductivity, these parts are defined 
by Michels and Trappeniers as kinetic term $\left(\kappa_{\mathrm{k}}\right)$, potential term $\left(\kappa_{\mathrm{p}}\right)$ and cross term $\left(\kappa_{\mathrm{c}}\right)$.

The unnormalized heat flux time correlation function of a hard-sphere fluid consists of a singular term s, which is entirely collisional part (cc) and a non-singular part $u$, which has kinetic part and a cross term (kc) and the remainder of the collisional part (cc). The nonsingular part of the heat flux relaxation function is

$$
C_{T}(t)=M_{\infty}^{\prime} \exp \left(-t / \tau_{\lambda}\right),
$$

where

$$
\frac{M_{\infty}^{\prime}}{k_{\mathrm{B}}}=\frac{5}{3} \rho k_{\mathrm{B}} T\left[1+\frac{3}{5}(Z-1)\right]^{2}
$$

and $\tau_{\lambda}=5 \sqrt{\pi} /[16(Z-1)]$ in hard-sphere reduced units.

The improved Enskog formula for the thermal conductivity of the pure hard sphere fluid, $\kappa$, is [13]:

$$
\frac{\kappa}{\kappa_{0}}=\frac{\rho b}{Z-1}\left[\left(1+\frac{3}{5}(Z-1)\right)^{2}+\frac{32}{25 \pi}(Z-1)^{2}\right],
$$

where $b=\left(2 \pi \sigma^{3} / 3\right)$ is second virial coefficient of the hard sphere fluid, $\mathrm{Z}$ is the compressibility factor defined as:

$$
Z=1+\frac{2}{3} \pi \rho^{*} g^{\mathrm{HS}}(\sigma),
$$

where $\rho^{*}$ is reduced density $\left(=\rho \sigma^{3}\right)$ and $g^{\mathrm{HS}}(\sigma)$ is the contact pair correlation function for hard-sphere fluid and can be calculated from the Carnahan and Starling [16] equation

$$
g^{\mathrm{HS}}(\sigma)=\frac{1-\eta / 2}{(1-\eta)^{3}},
$$

where $\eta=\pi \rho \sigma^{3} / 6$ is the packing fraction of hard sphere.

The value of the thermal conductivity in the limit of zero density, $\kappa_{0}$, from kinetic theory, is given by

$$
\frac{\kappa_{0}}{k_{\mathrm{B}}}=1.02513 \frac{75}{64 \sigma^{2}}\left(\frac{k_{\mathrm{B}} T}{m \pi}\right)^{1 / 2} \text {. }
$$

As in the Green-Kubo formula, the time correlation function of the hard-sphere fluid can be divided into singular and non-singular parts. The Enskog formula can also be divided into singular and non-singular parts of the thermal conductivity. The singular component of the thermal conductivity $\kappa^{\mathrm{S}}$ is

$$
\frac{\kappa^{\mathrm{S}}}{\kappa_{0}}=\frac{64}{75} \rho \sigma^{3}(Z-1)
$$

and the non-singular part $\kappa^{\mathrm{u}}$ is

$$
\frac{\kappa^{\mathrm{u}}}{\kappa_{0}}=\frac{\rho b}{Z-1}\left[1+\frac{3}{5}(Z-1)\right]^{2} .
$$

Up to Eq. (11), we have described the hard-sphere system. We have developed a method to calculate the transport properties of square-well system from a hard sphere system from Yu et al. work [17] (equation (3) of their paper). In this we have investigated that the transport property of a hard sphere system can be transformed into square-well system by replacing $g^{\mathrm{HS}}(\sigma)$, pair correlation function of hard sphere from $g^{\mathrm{SQ}}(\sigma)$, pair correlation function of square-well system in the expression of a particular transport property. This has been found correct for diffusion coefficient [14] and shear viscosity of the dense fluids [15]. Thus, thermal conductivity can be obtained by employing pair correlation function of square-well fluid. The sum of Eqs. (10) and (11) will give the same results after modification of $Z$.

Thus, the thermal conductivity for square-well fluid can be obtained by replacing pair correlation function $g^{\mathrm{HS}}(\sigma)$ in Eq. (7) by $g^{\mathrm{SQ}}(\sigma)$ as [17]:

$$
g^{\mathrm{SQ}}(\sigma)=g^{\mathrm{SW}}(\sigma)+\lambda^{2} g^{\mathrm{SW}}(\lambda \sigma) E \text {. }
$$

The $g^{\mathrm{SW}}(\sigma)$ and $g^{\mathrm{SW}}(\lambda \sigma)$ are the radial distribution functions evaluated at the points $\sigma$ and $\lambda \sigma$, respectively. The quantities $E$ and $g^{\mathrm{SW}}(\lambda \sigma)$ are defined as [17]

$$
E=\exp \left(\frac{\varepsilon}{k_{\mathrm{B}} T}\right)-\left(\frac{\varepsilon}{k_{\mathrm{B}} T}\right)-2 J
$$

where $J$ is the temperature dependent function as [17]

$$
J=\frac{0.5+0.28304 / T^{*}}{1+0.15360 / T^{*}}
$$

and

$$
g^{\mathrm{SW}}(\lambda \sigma)=g^{\mathrm{HS}}(\lambda \sigma) \exp \left(\frac{\alpha}{T^{*}}+\frac{\beta}{T^{* 2}}\right),
$$

where $T^{*}=k_{\mathrm{B}} T / \varepsilon$. The value of $\alpha$ and $\beta$ were determined to be -0.4317 and -0.1177 , respectively. The value of the radial distribution function for the hard-sphere fluid at $\lambda \sigma$ can be determined from the correlation by Monnery et al. [18]:

$$
g^{\mathrm{HS}}(\lambda \sigma)=0.99948+0.82404 \eta-3.46976 \eta^{2} .
$$

This correlation matches the Monte Carlo (MC) simulation results of Barker and Henderson [19] and is successfully applied in the calculation of diffusion coefficient by $\mathrm{Yu}$ and co-workers [17].

For square-well fluid, the pair correlation function $g^{\mathrm{SW}}(\sigma)$ can be written in high temperature approximation (HTA) [20] as

$$
g^{\mathrm{SW}}(\sigma)=g^{\mathrm{HS}}(\sigma)+\frac{1}{4 T^{*}} \frac{\partial a_{1}^{\mathrm{SW}}}{\partial \eta}+\frac{\lambda^{3}}{T^{*}} g^{\mathrm{HS}}(\lambda \sigma),
$$

where $a_{1}^{\mathrm{SW}}$ is the first order perturbation term associated with attractive energy $\varepsilon \phi$ and is given below [21]:

$$
a_{1}^{\mathrm{SW}}=-4 \eta\left(\lambda^{3}-1\right) \frac{1-\eta_{\mathrm{eff}} / 2}{\left(1-\eta_{\mathrm{eff}}\right)^{3}}
$$

and

$$
\eta_{\mathrm{eff}}=C_{1} \eta+C_{2} \eta^{2}+C_{3} \eta^{3}
$$

where the coefficients $C_{n}$ are given by matrix [21]:

$$
\begin{aligned}
& \left(\begin{array}{l}
C_{1} \\
C_{2} \\
C_{3}
\end{array}\right)= \\
& \left(\begin{array}{ccc}
2.25855 & -1.50349 & 0.249434 \\
-0.669270 & 1.40049 & -0.827739 \\
10.1576 & -15.0427 & 5.30827
\end{array}\right)\left(\begin{array}{l}
1 \\
\lambda \\
\lambda^{2}
\end{array}\right) .
\end{aligned}
$$

\section{Results and discussion}

The square-well fluid is one of the simplest ones possessing the basic characteristic of real fluids. It has 
proved to be the excellent model for a liquid as it holds the characteristic nature of hard-sphere collisions. Longuet-Higgest and Valleau [22] were first to use square-well model to describe the self-diffusion coefficients of dense fluids. Davis, Rice, and Sengers [23] developed a theory, called DRS theory analogously to the Enskog hard-sphere (EHS) theory for the calculations of transport coefficients of square-well fluids.

In the present work, we have calculated the thermal conductivity of square-well fluids employing the Enskog theory. Figure 1 shows the thermal conductivity for a square-well fluid as a function of reduced well-depth $\left(\varepsilon^{*}=1 / T^{*}\right)$ at various reduced densities. The numerical results presented in this paper are explained in the reduced units of thermal conductivity as described by Michels and Trappeniers [11]. It can be seen in Fig. 1 that the well-depth of the square-well fluid predicts a very small change in thermal conductivity where the change in diffusion coefficients and shear viscosity is larger [14, 15]. However, the change in the density of the fluid makes a significant change in the thermal conductivity of the square-well fluid. The present results are compared with the molecular dynamics results of the Michels and Trappeniers [11] at various well-depth $\left(\varepsilon^{*}\right)$ and densities. We find a good agreement between the present theoretical results and molecular dynamic results of Michels and Trappeniers. Michels and Trappeniers [11] have compared their molecular dynamic results with those predicted by DRS theory [23]. DRS theory predicts values sufficiently lower than molecular dynamic results of Michels and Trappeniers [11] at all densities. This means that the improved Enskog theory, applied in the present work, is much better than DRS theory. We find that the theoretical values obtained in the present work are higher than that of predicted molecular dynamic results [11].

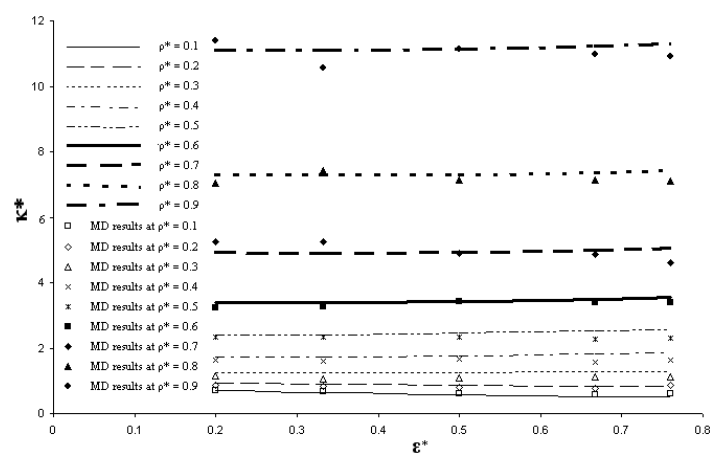

Fig. 1. The reduced thermal conductivity $\left(\kappa^{*}\right)$ of square-well fluid as the function of the reduced well depth $\left(\varepsilon^{*}\right)$ at various reduced densities $\left(\rho^{*}\right)$. Symbols are MD results of Michels and Trappeniers [11].

In the present work we present the radial distribution function at the point of contact of square-well fluid, i.e. the radial distribution function at the distance of the centers of the molecules at the moment of a collision for a square-well molecule. In the present work, this char-

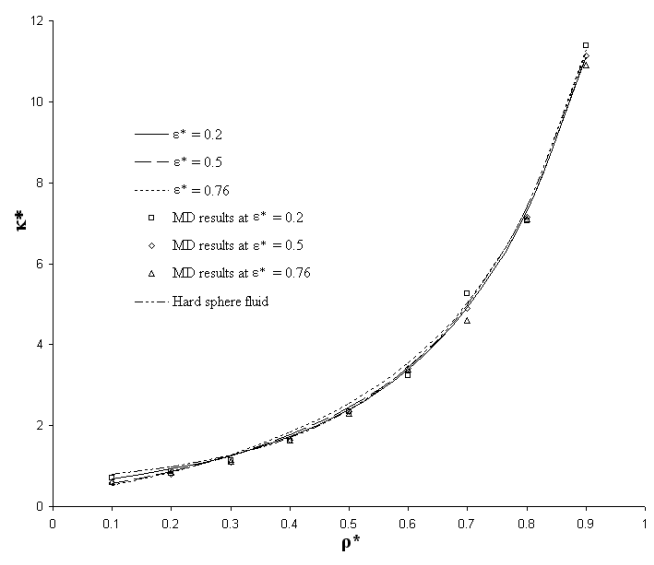

Fig. 2. The reduced thermal conductivity $\left(\kappa^{*}\right)$ for square-well fluid as the function of the reduced density $\left(\rho^{*}\right)$. The solid line is for $\varepsilon^{*}=0.2$, big dashed line for $\varepsilon^{*}=0.5$ and the small dashed line for $\varepsilon^{*}=0.76$, and the dashed dot dot line represents reduced thermal conductivity for hard-sphere fluid as the function of reduced density. Symbols are MD results of Michels and Trappeniers [11]. $\square$ for $\varepsilon^{*}=0.2, \diamond$ for $\varepsilon^{*}=0.5$ and $\triangle$ for $\varepsilon^{*}=0.76$.

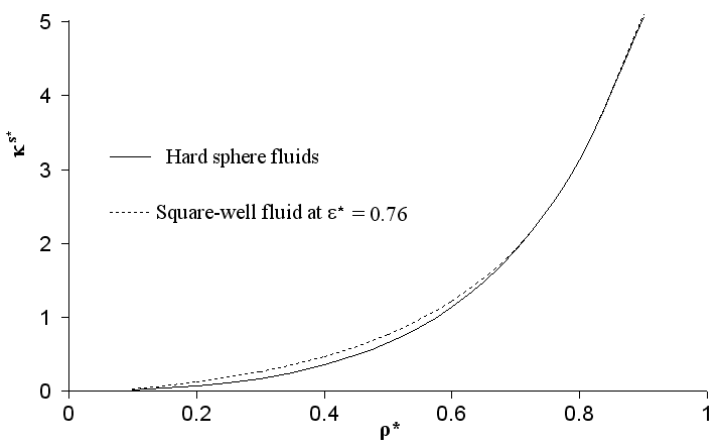

Fig. 3. The singular component of reduced thermal conductivity $\left(\kappa^{\mathrm{s}^{*}}\right)$ as the function of reduced density $\left(\rho^{*}\right)$. The solid line represents singular component of reduced thermal conductivity for hard-sphere fluid and the small dashed line represents the singular component of reduced thermal conductivity for square-well fluid at $\varepsilon^{*}=0.76$.

acteristic feature has been explored by calculating pair-correlation function at contact in high temperature approximation $[20,21]$. It appears that $\eta_{\text {eff }}$ employed in the calculations of the mean attractive energy plays an important role in the determination of thermal conductivity. This may be one reason that our results are better than DRS theory and it gives a good agreement with molecular dynamic results of Michels and Trappeniers [11].

Comparing the results with the hard-sphere system, it can be seen in Fig. 2 that the addition of an attractive part in the hard-sphere (square-well potential) is rather insensitive to the influence of the thermal conductivity. It is due to the fact that the kinetic, the potential and cross terms partially cancel each other. The addition 


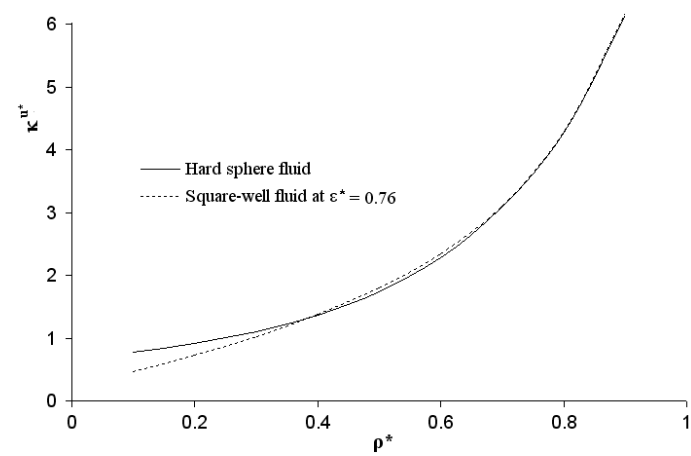

Fig. 4. The non-singular component of reduced thermal conductivity $\left(\kappa^{\mathrm{u}^{*}}\right)$ as the function of reduced density $\left(\rho^{*}\right)$. The solid line represents non-singular component of reduced thermal conductivity for hard-sphere fluid and the small dashed line represents the non-singular component of reduced thermal conductivity for square-well fluid at $\varepsilon^{*}=0.76$.

of attractive part on hard-sphere (square-well fluid) lowers the values of both the kinetic and cross term while the potential term increases with increasing well-depth. To show the insensitivity of attractive part in thermal conductivity, we have also calculated the singular and non-singular parts of thermal conductivity separately in Figs. 3 and 4, respectively. The singular part of square-well fluid remains higher than singular part of thermal conductivity of hard-sphere system at low and medium densities and ultimately merges into singular part of the hard-sphere system at high densities. Similarly, non-singular part of the square-well fluid remains lower than non-singular part of hard-sphere fluid at low densities and merges into non-singular part of thermal conductivity of the hard-sphere system at high densities. Thus both singular and non-singular parts of the thermal conductivity of square-well fluid remain the same as obtained in hard-sphere system at high densities while they are different at low and medium densities.

\section{Conclusion}

The study concludes that thermal conductivity is a property of liquids which does not depend on reference systems such as HS and square-well while other properties such as viscosity and diffusion coefficients depend on reference system.

\section{Acknowledgments}

Authors are thankful to C.S.I.R. (New Delhi) for financial assistance.

\section{References}

[1] S. Chapman, T.G. Cowling, The Mathematical Theory of Non-Uniform Gases, Cambridge University Press, Cambridge 1990.

[2] U. Balucani, M. Zoppi, Dynamics of Liquid State, Clarendon, Oxford 1994.

[3] J. Bosse, W. Gotze, M. Lucke, Phys. Rev. A 17, 434 (1978).

[4] E. Leutheusser, J. Phys. C 15, 2801 (1982).

[5] D.J. Evans, Phys. Lett. A 91, 457 (1982).

[6] D.M. Heyes, J. Chem. Soc. Faraday Trans. 2 80, 1363 (1984).

[7] D.M. Heyes, J. Phys., Condens. Matter 6, 6409 (1994).

[8] R.K. Sharma, K. Tankeswar, K.N. Pathak, J. Phys., Condens. Matter 7, 537 (1995).

[9] A.E. Nasrabad, R. Laghaei, B.C. Eu, J. Chem. Phys. 124, 084506 (2006).

[10] D.M. Heyes, J.G. Powles, Mol. Phys. 99, 1077 (2001).

[11] J.P.J. Michels, N.J. Trappeniers, Physica A 107, 158 (1981).

[12] A.C. Branka, D.M. Heyes, Phys. Rev. E 69, 021202 (2004).

[13] P. Resibois, M. de Leener, Classical Kinetic Theory of Fluids, Wiley, New York 1977, p. 168.

[14] D.K. Dwivedee, R. Srivastava, K.N. Khanna, Fluid Phase Equilibria 263, 199 (2008).

[15] R. Srivastava, A. Tewari, K.N. Khanna, Indian J. Pure Appl. Phys. 47, 568 (2009).

[16] N.F. Carnahan, K.E. Starling, J. Chem. Phys. 51, 635 (1969).

[17] Y.X. Yu, M.H. Han, G.H. Gao, Phys. Chem. Chem. Phys. 3, 437 (2001).

[18] W.D. Monnery, W.Y. Svrcek, A.K. Meherotra, Fluid Phase Equilibria 117, 378 (1996).

[19] J.A. Barker, D. Henderson, Mol. Phys. 21, 187 (1971).

[20] M. Banaszak, Y.C. Chiew, M. Radsoz, Phys. Rev. E 48, 3760 (1993).

[21] A. Gil-Villegas, A. Galindo, P.J. Whitehead, S.J. Mills, G. Jackson, J. Chem. Phys. 106, 4168 (1997).

[22] H.C. Longuet-Higgins, J.P. Valleau, Mol. Phys. 1, 284 (1958).

[23] H.T. Davis, S.A. Rice, J.V. Sengers, J. Chem. Phys. 35, 2210 (1961). 